\title{
Comparison between time and frequency studies of a corrugated curve of RER Paris network
}

\author{
C. Collette ${ }^{\mathrm{a}, *}$, P. Vanhonacker ${ }^{\mathrm{b}}$, R. Bastaits ${ }^{\mathrm{a}}$, D. Levy ${ }^{\mathrm{c}}$ \\ a University of Brussels, Department of Mechanical Engineering and Robotics, \\ Active Structures Laboratory, 50 av. F.D. Roosevelt, 1050 Brussels, Belgium \\ ${ }^{b}$ D2S International, 71 Jules Vandenbemptlaan, 3001 Heverlee, Belgium \\ ${ }^{c}$ RATP, 50 rue Roger Salengro, 94724 Fontenay-sous-bois Cedex, France
}

\section{A R T I C L E I N F O}

\section{Article history:}

Accepted 21 January 2008

Available online 20 May 2008

\section{Keywords:}

Rail corrugation

Multi-body model

Wheel-rail contact

Wear

On-site measurements

\begin{abstract}
A B S T R A C T
In this paper, both time and frequency domain approaches are applied to a corrugated, curved, ballasted track section located in the RER commuter rail network in Paris. From the railhead profile measurements, two corrugation wavelengths are identified on the low rail. The wavelength fixing mechanisms are found to correlate to a resonance of the concrete sleepers and a resonance of the unsprung mass of the vehicle on the track stiffness. Corrugation growth rates are established from both approaches and compared. Then, an extensive parameter study is performed in order to evaluate the relevance of various parameters and to identify viable solutions for mitigating the observed corrugation. Specifically we examine the impact on rail corrugation due to the following track parameters: the vertical and lateral rail pad stiffness, the sleeper mass and the superelevation.
\end{abstract}

(c) 2008 C. Collette. Published by Elsevier B.V. All rights reserved.

\section{Introduction}

During the last 20 years, different approaches for the study of rail corrugation have been developed, these can be classified in two categories: frequency domain corrugation theories and time domain non-linear models. Although numerous publications examine these techniques the literature, few comparisons have been published.

In this paper, both time and frequency domain approaches are applied to a corrugated, curved, ballasted track section located in the RER commuter rail network in Paris. From the railhead profile measurements, two corrugation wavelengths are identified on the low rail.

The method used for the measurements and descriptions of the case studied are detailed in next section. Then, from both frequency and time approaches, the wavelength fixing mechanisms are found to correlate to a resonance of the concrete sleepers and a resonance of the unsprung mass of the vehicle. Prediction similarities and differences are discussed from the longitudinal and the lateral corrugation growth coefficients.

Finally, a sensitivity analysis is performed on a few parameters of the system in order to investigate their impact on the development of corrugation.

\footnotetext{
* Corresponding author. Tel.: +32 265046 62; fax: +32 26504660 E-mail address: christophe.collette@ulb.ac.be (C. Collette).

URL: http://www.ulb.ac.be/scmero (C. Collette).
}

\section{Case description}

\subsection{Background}

The line B of the RER network of the RATP uses in its southern part an old railway line called "Ligne de Sceaux". The Ligne de Sceaux was inaugurated in 1846 to experiment the "Arnoux" principle of articulated railroad vehicle with directional wheel sets, allowing trains to run in curves up to $30 \mathrm{~m}$ radius.

Another interesting point is that the track has been built with a large gauge of $1.75 \mathrm{~m}$. In 1891 the rail spacing was changed to the standard value of the national network to allow other vehicles to run on this track.

Many curves with small radius still exist from these early line drawings, which are sections where corrugation is prone to develop, and even more specifically on rails of its branch "Robinson".

\subsection{Context}

Without any specific adapted measure, long welded rails are not suited for curves with a small radius. For this reason, rails of the track section considered for this study had been linked together with in-between joints until 2002. When the track and ballast have been renewed (RVB), it was decided to weld the rails. For this reason, the track was reinforced, wooden sleepers were replaced by concrete sleepers, the rail weight was decreased from 60 (60E1) to 
$50 \mathrm{~kg} / \mathrm{m}$ (50E6) and anchoring devices were fixed under the sleepers to ensure the transverse stability of the track. Only geometrical characteristics of the track remained unchanged.

After the RVB was carried out on the line "Robinson", severe corrugation appeared on the curves, with a very high speed of reappearance. As a consequence, damages of the rail tread provoked an important increase of the emitted noise, involving numerous complaints from the residents.

Before the RVB, rail corrugation was also visible on the rails. However, the frequency of grindings carried out before and the absence of complaints let the authors suppose that it was growing very slowly, even if there are unfortunately no systematic measurements available to attest it.

In order to understand this difference in behavior, the Track Department of the RATP asked the Active Structures Laboratory from the University of Brussels (also member of the European research project CORRUGATION) to perform a numerical study to identify relevant track parameters responsible for corrugation to develop, using a multi-body model of the whole vehicle track system.

\subsection{Corrugation characterization}

The track section chosen for the study is a curve located at the exit of the station "Fontenay aux Roses" with a radius of $250 \mathrm{~m}$. The line drawing is depicted in Fig. 1.
The axle load of the rolling stock is $18 \mathrm{~T}$, and the maximum speed of the vehicle is $55 \mathrm{~km} / \mathrm{h}$. On track 1Bis, vehicles are accelerating as they leave the station, while on track 2 Bis vehicles are braking as they enter the station. The axle load allowed is 27,800 T/d.

Rail corrugation appears only on the low rail of the track. The wavelength is varying between 8 and $20 \mathrm{~cm}$, with a dominant component around $16 \mathrm{~cm}$ often superimposed on a wave of lower amplitude of $8 \mathrm{~cm}$ (Fig. 2). Its growth is very fast: after 3-4 weeks, it reaches a depth of $0.6 \mathrm{~mm}$.

The contact surface of the rail is different on the crest and in the troughs. The crests show a smooth surface, while the troughs show much more irregularities, including a lot of micro-holes corresponding to particle detachment (Fig. 3).

The orientation of these holes towards the outside of the track shows that the particles were ejected in this direction. Tribological studies showed that the holes result from lateral material flow towards the inside of the curve, which involve an excessive wear of these zones.

\subsection{Measurements and follow-up of corrugation growth}

Rail corrugation was measured using a specific trolley, designed by the RATP, whose basic principle is to measure the depth of the undulation from a reference line sliding on the rail surface in the longitudinal direction. The measuring part of the carriage is made

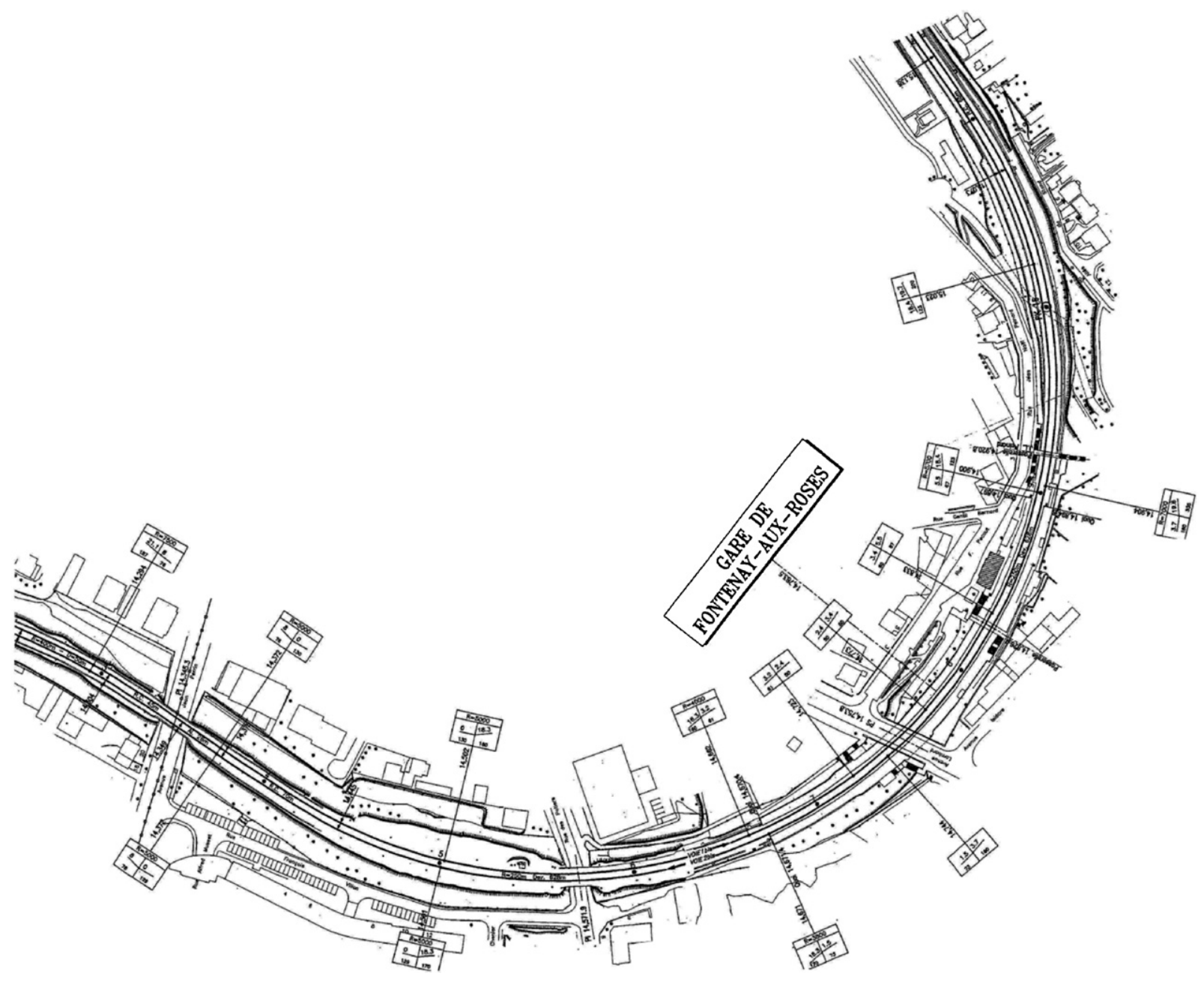

Fig. 1. Line drawing [1]. 


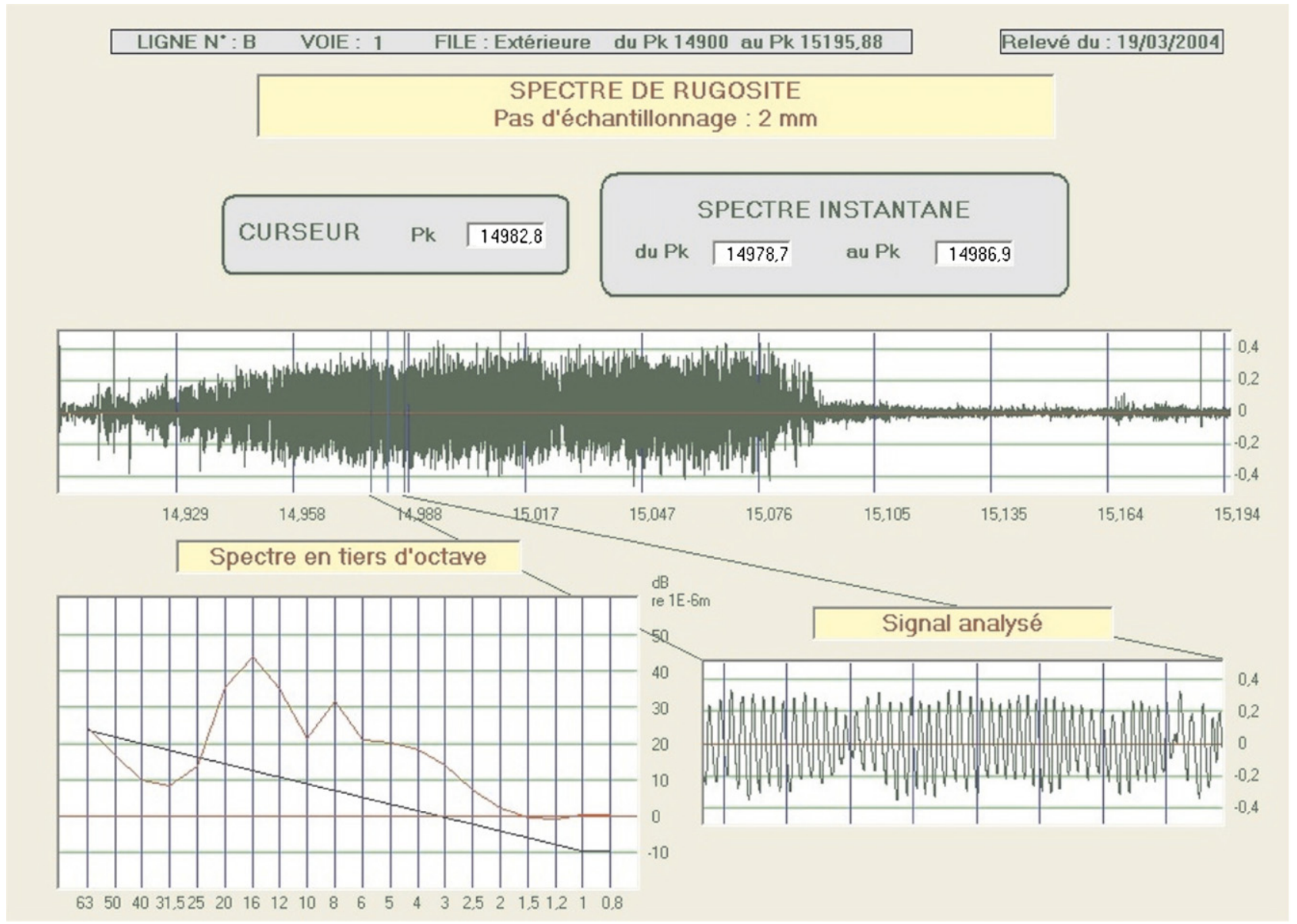

Fig. 2. Roughness spectrum [1].

of two skates of $80 \mathrm{~cm}$ length, one sliding on each rail treads, and both mounted with a non-contact Eddy current displacement sensor. Measurements are performed on the two rails simultaneously (Fig. 4). After acquisition, measurement data are post-treated in accordance with the standard prEN 13231-3 [2].

Up to now, the curve is ground as often as possible in order to disturb the least possible the residents living in the vicinity with the induced vibrations and the noise.

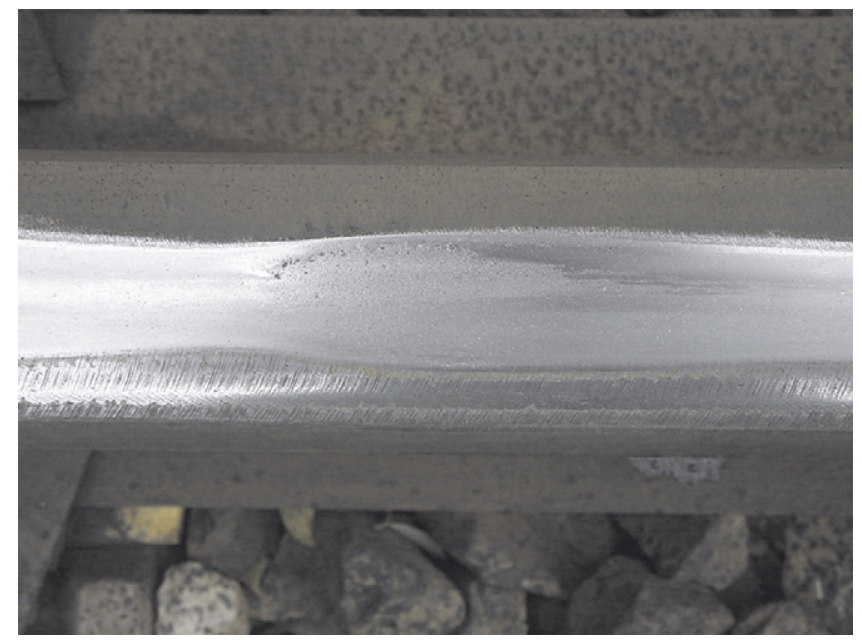

Fig. 3. Picture of corrugation [1].
In Bilbao, a similar problem has been treated by changing the rail pad stiffness, and led to encouraging results. Following these studies, rail pads of three different stiffnesses have been randomly laid out in June 2004 in order to modify the dynamic response of the track. The results are given in terms of the extension of rail corrugation along the track (Fig. 5) and by the follow-up of the evolution of its maximum amplitude (Fig. 6).

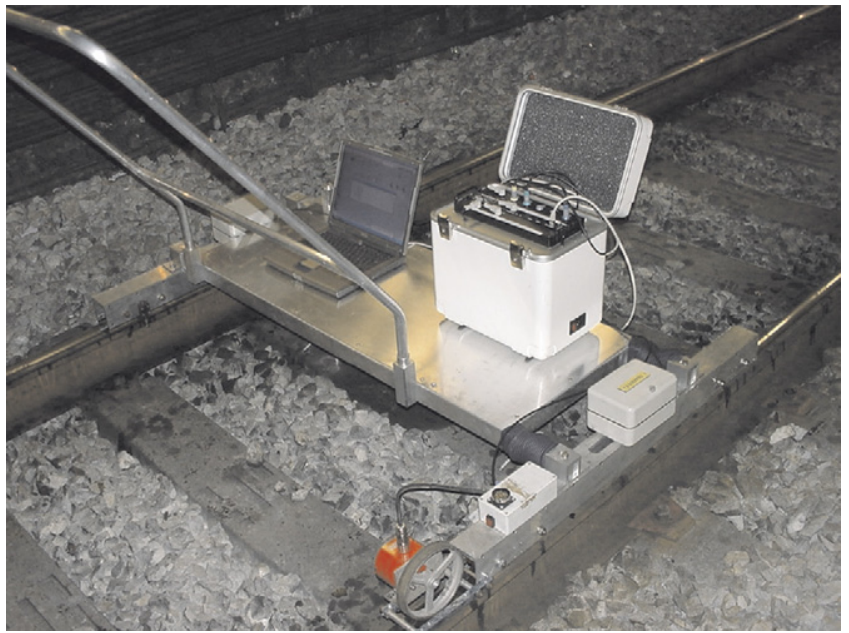

Fig. 4. Trolley for the measurement of rail corrugation [1]. 


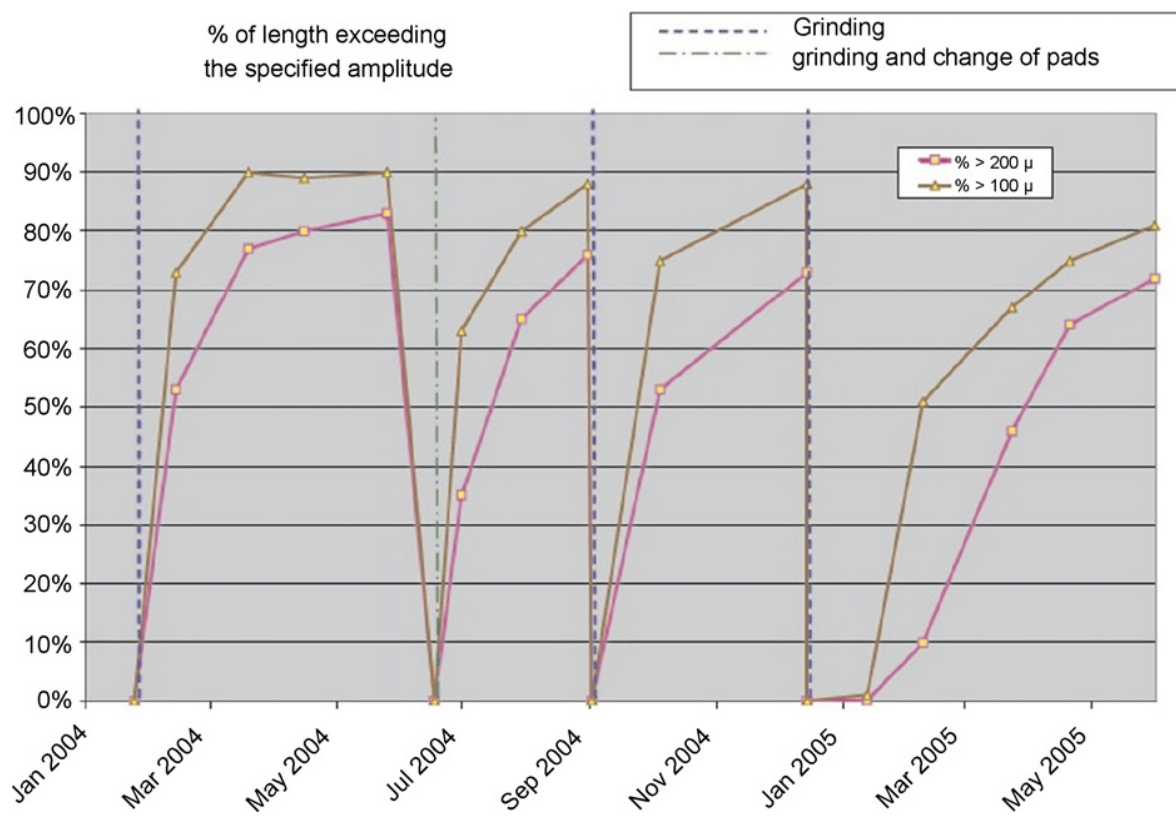

Fig. 5. Corrugation extension after grinding [1].

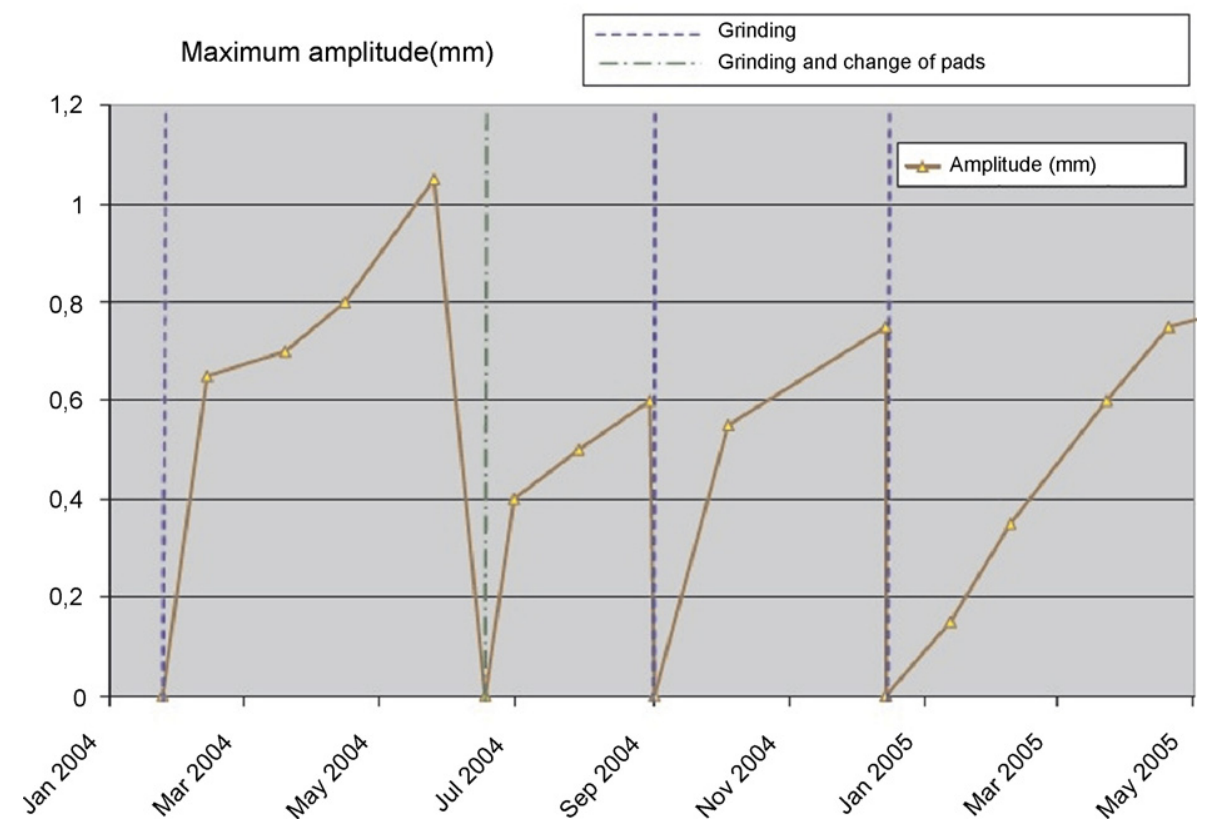

Fig. 6. Corrugation growth after grinding [1].

Rail corrugation grows very fast, with a high amplitude, to reach the whole curve in a couple of weeks time. The replacement of rail pad has no significant impact on the speed of appearance; only the growth is slightly less fast. However, with improvements in the quality of grinding, such as to realize extra grinding after the removal of the visible corrugation (January 2005), it is possible to delay a little ( 3 weeks) the appearance of corrugation.

\section{Frequency domain}

\subsection{A literature review}

The first linear theory of rail corrugation has been presented in Ref. [3]. A successful application of this theory can be found in Refs.
[4-6] to explain the rail corrugation in Bilbao appearing between sleepers. Calculations are very fast, and the theory can benefit from a range of tools developed for the study of linear systems, like stability analysis [7]. Since its first formulation [3], a few authors proposed improvements for the calculation of the wear rate. For instance, in [8,9], an exponential phenomenological law between the creepage and the creep force has been introduced in the theory, as well as a vertical-lateral cross receptance of the vehicle and the track to account for the coupling between the two directions. Then, contact filters have been introduced in [10] to account for the finite size of the contact patch. This lead to a better representation of the corrugation growth rate for short pitch corrugation. A geometry constant mechanism has been introduced in [11,12] to explain the non-dependency of short pitch corrugation wavelength 
with the vehicle speed. More recently, a modal decomposition of the vehicle track dynamic equations has been introduced in [13]. Accordingly, they were able to propose analytical formulations of the corrugation growth rate for each mode of the system.

The model used for the study is restricted to the basic approach of $[8,9]$, sufficient for the description of the wavelength fixing mechanism of corrugation phenomena of a wavelength above $5 \mathrm{~cm}$. The model is presented in next section.

\subsection{The linearity assumption}

The vehicle-track system includes geometrical non-linearities (wheel and rail profiles) and physical non-linearities (relationship between the contact load and the associated bodies deformations, relationship between the creepage and the associated creep force). In order to study the system in the frequency domain, all the equations must be linearized around a constant value. In other words, for each quantity $q$, only small variations $\Delta q$ around a reference value $q_{0}$ are considered.

\subsection{Description of the model}

After each passage of the wheel on the rail, the rail profile is slightly modified by the wheel-rail contact creep forces. According to [14], the mass $\Delta m$ removed from the rail surface after the $n^{\text {ieme }}$ wheel passage is proportional to the frictional work $\Delta W$ of the creep forces

$\frac{\partial \Delta m(\omega, n)}{\partial n}=k_{0} \Delta W(\omega, n)$

where $k_{0}$ is a material related coefficient and $\omega$ is the circular frequency, related to the vehicle speed $V$ and to the wavelength of the irregularity $\lambda$ by $\omega=2 \pi V / \lambda$. Assuming that the mass $\Delta m$ of a volume $\Delta x \Delta y \Delta z$ is removed from the rail surface at a speed $V$ and that its width is equal to the width of the contact patch $(\Delta y=2 b)$ Eq. (1) can be rewritten as:

$\frac{\partial \Delta z(\omega, n)}{\partial n}=-\frac{k_{0}}{2 \rho b V} \Delta P(\omega, n)$

where $\rho$ is the density, $\Delta P=\Delta W / \Delta t$ is the frictional power dissipated in the contact and $\Delta t=\Delta x / V$. Without any lateral creep, i.e. $v_{y}=0$,

$\Delta P=\Delta P_{x}=V\left(\Delta v_{x} F_{x}+v_{x} \Delta F_{X}\right)$.

Without any longitudinal creep, i.e. $v_{x}=0$,

$\Delta P=\Delta P_{y}=V\left(\Delta v_{y} F_{y}+v_{y} \Delta F_{y}\right)$.

Using $\Delta P_{x}$ and $\Delta P_{y}$, two corrugation growth coefficients $G_{x}$ and $G_{y}$ can be defined as

$\left[\begin{array}{l}G_{x} \\ G_{y}\end{array}\right]=-\frac{k_{0}}{2 \rho b V \Delta z(\omega, n)}\left[\begin{array}{l}\Delta P_{x} \\ \Delta P_{y}\end{array}\right]$

Most of the time, wear results from a longitudinal and a lateral friction. Using Eqs. (3)-(5), Eq. (2) can be rewritten as

$\frac{\partial \Delta z(\omega, n)}{\partial n}=G(\omega) \Delta z(\omega, n)$

and $G(\omega)=G_{x}(\omega)+G_{y}(\omega)$. Eq. (6) governs the time evolution of the rail profile. Analytical formulations of $G_{x}(\omega)$ and $G_{y}(\omega)$ are given in Appendix A.

The corrugation growth rate, also called the corrugation local eigen value, is given by the real part of $G(\omega)$. Positive values of $\Re\{G(\omega)\}$ correspond to wavelengths that will be magnified; negative values of $\Re\{G(\omega)\}$ correspond to wavelengths that will be flattened. The higher the value of $\Re\{G(\omega)\}$, the faster corrugation will develop. The general procedure to evaluate the corrugation growth coefficient in the frequency domain is shown in Fig. 7.

\subsection{Application to the test case}

A mean wear rate has been estimated from Figs. 5 and 6 at $G=$ $4.5 \times 10^{-5}$. This value has been obtained as follows. The load per day on the track is $27,800 \mathrm{~T}$, the axle load is $18 \mathrm{~T}$, the number of wheel passing on each rail every day is $27,800 / 18$. From the Fig. 6 , since the quality of the grinding has been improved, the amplitude of corrugation is about $0.6 \mathrm{~mm}$ after two months and a half. Assuming an initial roughness of $4 \mu \mathrm{m}$ peak and an exponential evolution, it leads to a growth factor of $G=4.5 \times 10^{-5}$.

The reference values of the parameters used for the study are: $V=15 \mathrm{~m} / \mathrm{s} ; v_{x}=0.003 ; v_{y}=0.01 ; N=88,000 \mathrm{~N}$. Lateral and vertical vehicle and track receptances have been measured by hammer tests and are shown respectively in Figs. 8 and 9. In the lateral direction, the peak in the lateral receptance around $180 \mathrm{~Hz}$ (Fig. 8)

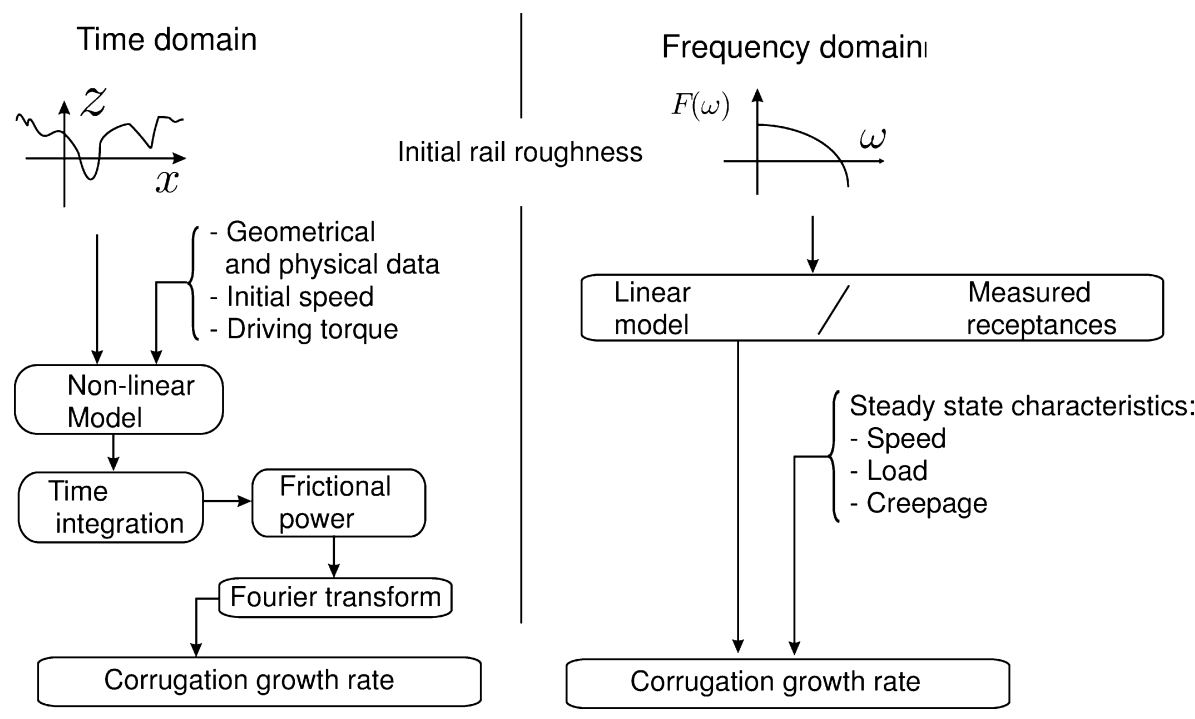

Fig. 7. General procedure for the evaluation of corrugation growth rate in the time and frequency domain. 


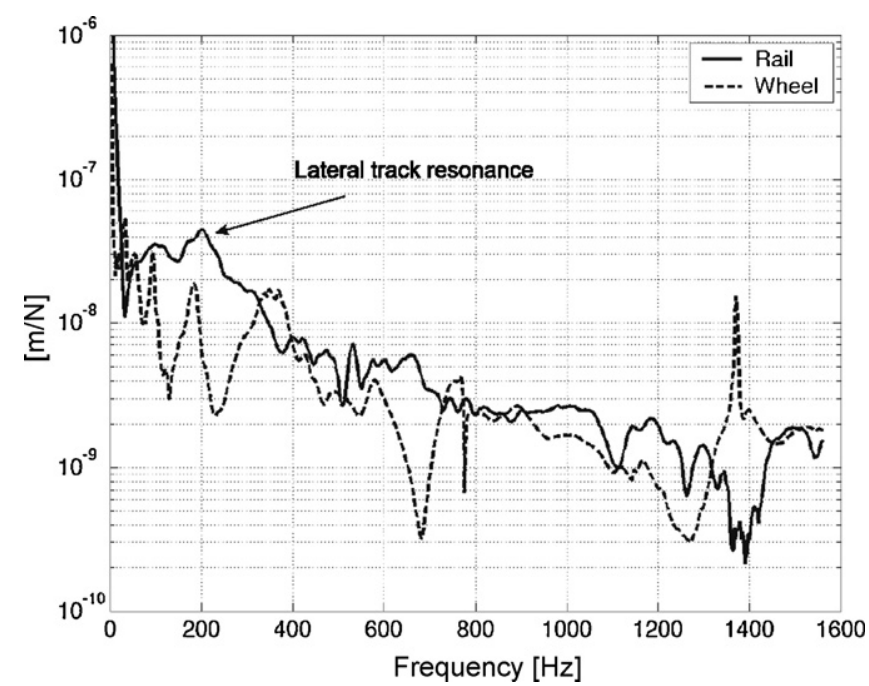

Fig. 8. Measured lateral vehicle and track receptances.

corresponds to a resonance of the sleeper, vibrating out of phase with the rails. In the vertical direction, the minimum in the vertical receptance around $180 \mathrm{~Hz}$ (Fig. 9) corresponds to an anti-resonance of the track. At that frequency, the sleeper is vibrating while the rail is not vibrating at all.

Using the measured receptances and the steady-state conditions, the longitudinal and lateral corrugation growth rates have been calculated by Eqs. (9) and (10) and are shown in Fig. 10.

The corrugation growth rate in the longitudinal direction is one order of magnitude smaller than the corrugation growth rate in the lateral direction. Regarding lateral direction, a range between 80 and $200 \mathrm{~Hz}$ is positive. In this range, two peaks are visible in Fig. 10. The first one, at $90 \mathrm{~Hz}$, is associated to the wavelength of $16 \mathrm{~cm}$. The wavelength fixing mechanism is recognized to be the so-called $\mathrm{P} 2$ resonance [15], i.e. a resonance of the unsprung mass of the vehicle on the track stiffness. This resonance creates high variations of the vertical contact force, responsible for corrugation.

The second one, around $180 \mathrm{~Hz}$, is associated to the wavelength of $8 \mathrm{~cm}$. Due to the resonance of the sleeper (Figs. 8 and 9), the initial rail roughness creates important variations of the normal contact force whose engender simultaneous variations of the creep forces responsible for limit cycles in the creepage-creep force

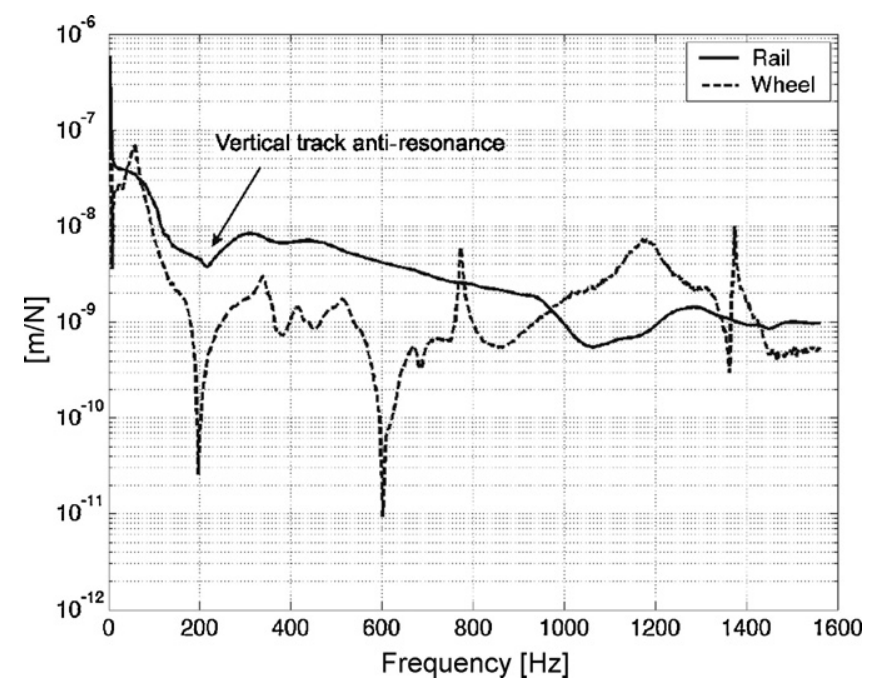

Fig. 9. Measured vertical vehicle and track receptances.

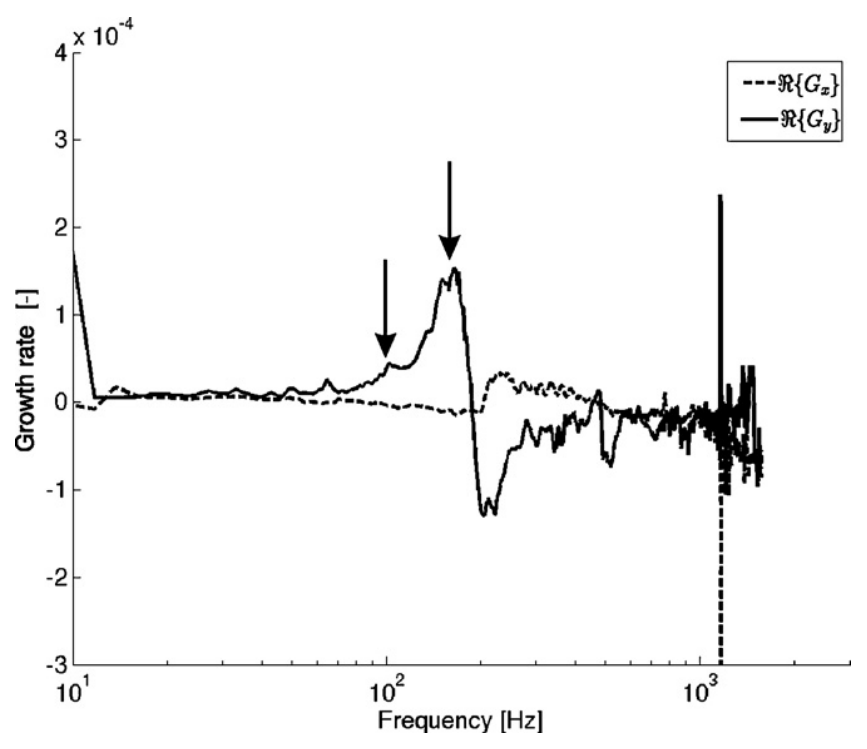

Fig. 10. Corrugation growth rates calculated from measurements.

phase plane, and accordingly for corrugation to develop. The phenomenon is exacerbated by a lateral resonance of the track around the same frequency. This type of corrugation can be classified in the category of booted sleeper corrugation in [15]. In that paper, the wavelength associated to this type of corrugation is in the range between 45 and $60 \mathrm{~mm}$, in stead of $8 \mathrm{~cm}$ in this case. The main reason for this difference is that the sleeper resonance (in vertical and lateral direction) is around $180 \mathrm{~Hz}$ in this case and between 300 and $500 \mathrm{~Hz}$ in the case of subway tracks. A drawing of the formation of the two types of corrugation is presented in Fig. 11.

Similar test cases with two corrugation patterns have already been reported in the literatures $[8,16]$. For each of them, the first peak is smaller than the second one like it is also the case in this study. However, from measurements, the opposite situation has been recorded: the wavelength of $16 \mathrm{~cm}$ related to a frequency of $90 \mathrm{~Hz}$ corresponds to the rail corrugation with the highest amplitude (see Fig. 2). The vertical and lateral track receptances have been measured above a sleeper and between two sleepers. Below $400 \mathrm{~Hz}$, no significant difference was noticed by the authors between the two measurements.

\section{Time domain}

\subsection{A literature review}

In the time domain approach any type of non-linearities can be taken into account in the integration. Due to the huge integration time, first models were restricted to vertical dynamics [17], to become more and more complicated in recent works [18-20,22]. For the same reason of integration time, contact models used in time domain simulations of corrugation are stationary. Time dependant formulations like [21-23] are useful only if the high frequency dynamics of the vehicle and the track are also included in the model.

\subsection{Description of the model}

In the time domain, the wear is evaluated using the same assumption that the mass $\Delta m$ removed from the rail surface after the $n$th wheel passage is proportional to the work $\Delta W$ of the creep forces between the two bodies. In this case however, the new rail profile obtained after the passage of the wheel is directly evaluated 


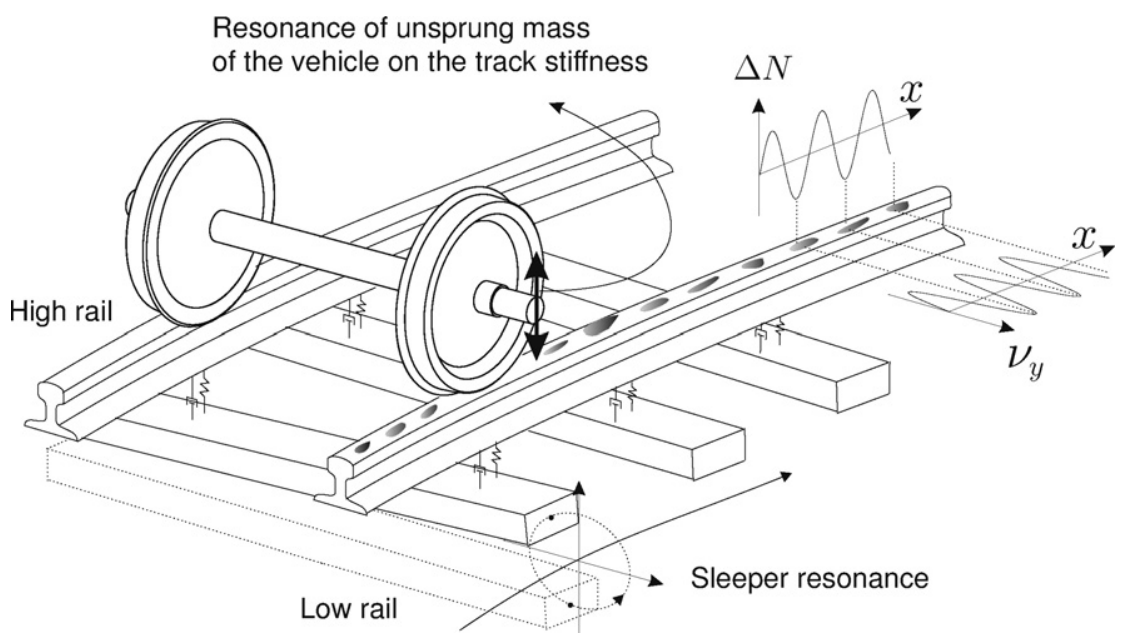

Fig. 11. Formation mechanism of corrugation studied in the test case.

from the calculation of the time history of the friction power dissipated in the contact patch, and Eq. (2). In order to compare the results with those from the frequency domain study, Eqs. (9) and (10) can be written as:

$$
\left[\begin{array}{l}
G_{x} \\
G_{y}
\end{array}\right]=-\frac{k_{0}}{2 \rho b V \Delta z(\omega, 0)}\left[\begin{array}{c}
\Delta P_{x} \\
\Delta P_{y}
\end{array}\right]
$$

where $\Delta P_{x} \Delta P_{y}$ are the power spectral densities of the frictional power dissipated in the longitudinal and lateral direction, $\Delta z(\omega, 0)$ is the initial irregularity.

The general procedure to evaluate the corrugation growth coefficient in the time domain is also shown in Fig. 7.

\subsection{Application to the test case}

A complete multi-body model of the vehicle-track system has been developed for portraying the vehicle motion on the track using the software SIMPACK [24] and is depicted on Fig. 12. The model includes a two-layered track as well as a finite element representation of the wheel-set. Rails and sleeper are rigid bodies that follow each wheel set above them. The wheel-rail creep forces are calculated using the approximation of Polach [25] in dry contact condition. This model includes negative slope of relationship between the creepage and the creep force after the saturation that able to take into account non-linear phenomena like stick-slip.

Starting from the data set of physical and geometrical quantities of the vehicle-track system, given by the RATP, unknown track parameters are calibrated in order that vertical and lateral track receptances fit the measured ones. Table 1 summarizes the main vehicle and track characteristics of the case studied.

For comparison, the linear theory has been applied to the multibody model and shown in Fig. 13. Three peaks are visible on the figure : the first spread one around $90 \mathrm{~Hz}$ corresponds to the P2 resonance, the second sharper one corresponds to a contribution of the second bending mode of the wheel set, and the third one at $180 \mathrm{~Hz}$ is associated to a vertical resonance of the sleeper. This figure shows a consistency with the results obtained from the direct application of the method on the measured data (see Fig. 10). Additionally, a clear distinction between the first and the second corrugation pattern is now visible in Fig. 13.

For the simulation, the initial roughness spectrum is taken from the standard Simpack library

$Z_{0}(\omega)=\frac{0.001518}{0.016986+0.8452 \omega+\omega^{2}}$ and $\omega=2 \pi / \lambda$ and is shown in Fig. 14(a). During the simulation, creepages and creep forces are calculated at each time step in the longitudinal and the lateral direction. Fig. 14(b)-(e) shows the time histories of these quantities for the front bogie.

For comparison with the linear theory, corrugation growth rates are calculated using Eq. (7) and are shown in Figs. 15 and 16. Only corrugation growth rates of the inner wheel of the front wheel-set are discussed, because it is on this wheelset that the highest lateral forces are applied.

From Figs. 15 and 16, we see that the ratio between the first and the second peak is higher than the result obtained with the frequency approach, and much closer to the measurements (Fig. 2). An explanation for this better agreement is given by the basic

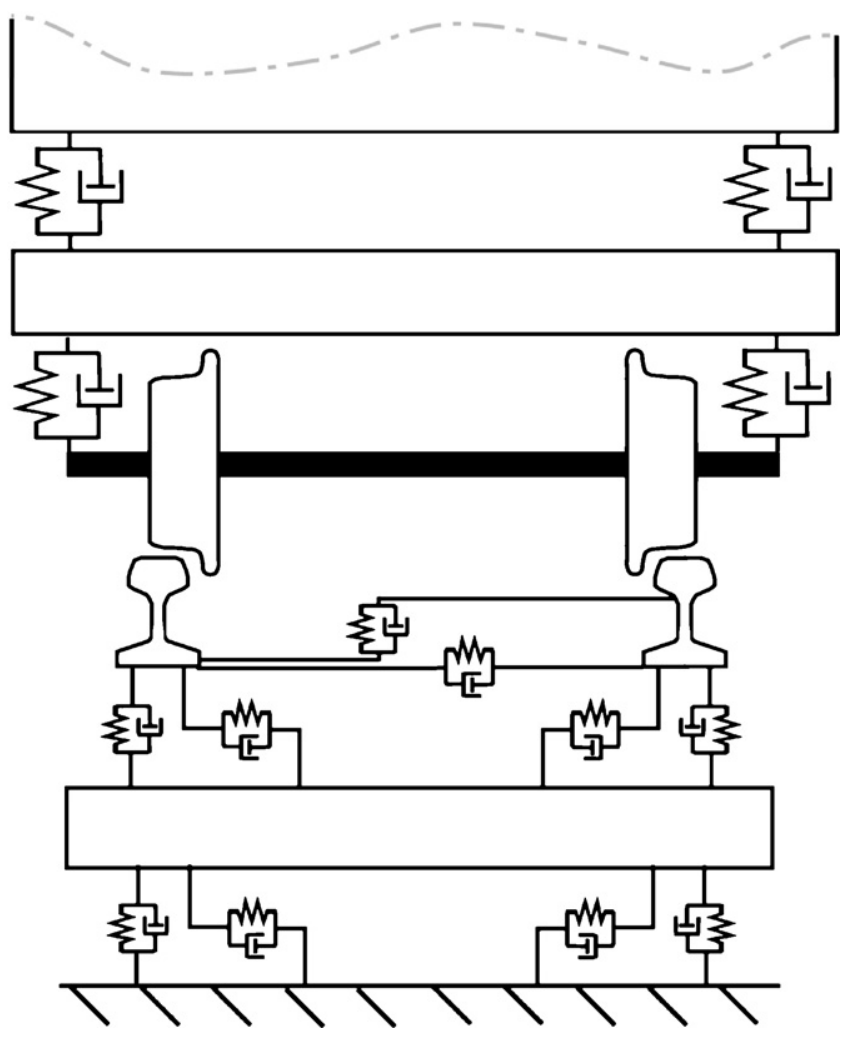

Fig. 12. Front view of the vehicle-track multi-body model. 
Table 1

Vehicle-track parameters

\begin{tabular}{lll}
\hline Vehicle parameters & & Value \\
\hline Car body mass (loaded) & & $46,850 \mathrm{~kg}$ \\
Bogie mass & & $8520 \mathrm{~kg}$ \\
Unsprung mass & & $2100 \mathrm{~kg}$ \\
Wheel radius & & $0.510 \mathrm{~m}$ \\
Train speed & & $15 \mathrm{~m} / \mathrm{s}$ \\
Primary suspension & Longitudinal stiffness & $1.9 \mathrm{E} 6 \mathrm{~N} / \mathrm{m}$ \\
& Lateral stiffness & $1.4 \mathrm{E} 6 \mathrm{~N} / \mathrm{m}$ \\
& Vertical stiffness & $1.2 \mathrm{E} 6 \mathrm{~N} / \mathrm{m}$ \\
& Longitudinal damping & $25 \mathrm{E} 3 \mathrm{Ns} / \mathrm{m}$ \\
& Lateral damping & $5 \mathrm{E} 3 \mathrm{Ns} / \mathrm{m}$ \\
& Vertical damping & $5 \mathrm{E} 3 \mathrm{Ns} / \mathrm{m}$ \\
Secondary suspension & Longitudinal stiffness & $292 \mathrm{E} 3 \mathrm{~N} / \mathrm{m}$ \\
& Lateral stiffness & $292 \mathrm{E} 3 \mathrm{~N} / \mathrm{m}$ \\
& Vertical stiffness & $556 \mathrm{E} 3 \mathrm{~N} / \mathrm{m}$ \\
& Longitudinal damping & $8 \mathrm{E} 3 \mathrm{Ns} / \mathrm{m}$ \\
& Lateral damping & $8 \mathrm{E} 3 \mathrm{Ns} / \mathrm{m}$ \\
& Vertical damping & $8 \mathrm{E} 3 \mathrm{Ns} / \mathrm{m}$ \\
Track parameters & & Value \\
U50 rail & Mass (length=1.25 m) & $62.5 \mathrm{~kg}$ \\
Sleeper & Mass & $310 \mathrm{~kg}$ \\
Rail pad & Stiffness (lateral/vertical) & $62 / 175 \mathrm{E} 6 \mathrm{~N} / \mathrm{m}$ \\
Ballast & Damping (lateral/vertical) & $5 / 10 \mathrm{E} 3 \mathrm{Ns} / \mathrm{m}$ \\
& Stiffness (lateral/vertical) & $23 / 35 \mathrm{E} 6 \mathrm{~N} / \mathrm{m}$ \\
& Damping (lateral/vertical) & $90 / 38 \mathrm{E} 3 \mathrm{Ns} / \mathrm{m}$ \\
\hline & & \\
& &
\end{tabular}

difference of input in the two models. In the frequency domain, the corrugation growth coefficients are evaluated from the vehicle and track receptances obtained with input random force or displacements. However, in the time domain, the corrugation growth coefficients are evaluated from initial rail irregularities, that are far from randomness, and lead to a higher excitation of the low frequency modes than the higher frequency ones. As a consequence, because of the geometrical and physical non-linearities

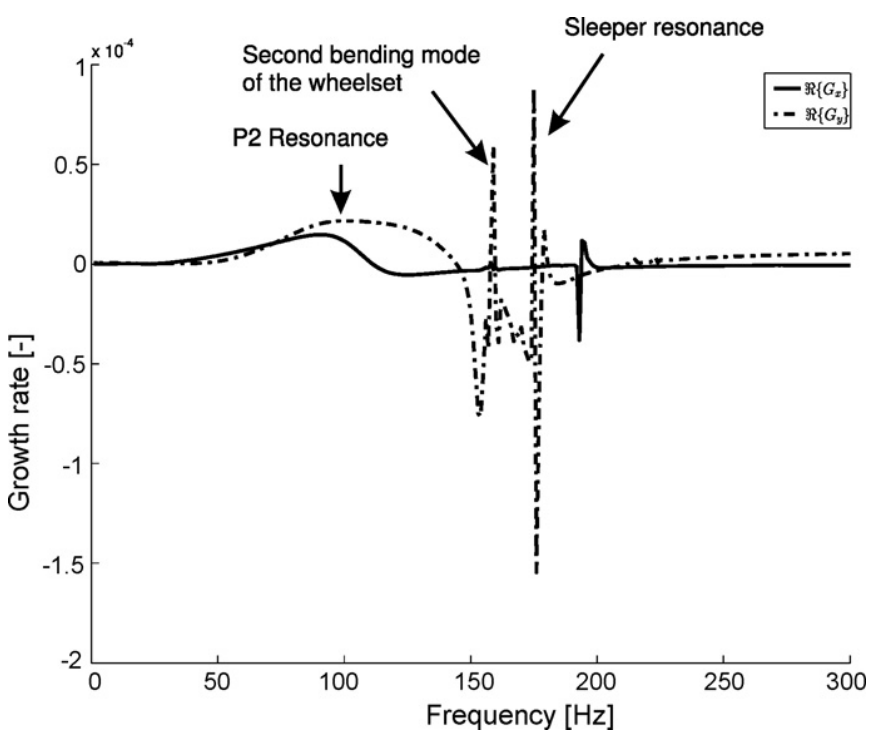

Fig. 13. Corrugation growth rates calculated by the linear theory using receptances of the multi-body model.

taken into account in the time domain approach, results are not the same.

\section{Parameter variations}

In this section, a parameter study is performed in order to evaluate the relevance of various parameters in corrugation growth and to identify viable solutions for mitigating the observed corrugation. Parameter investigated and their influence on the predicted corrugation growth rates are listed in Table 2. Only results for the
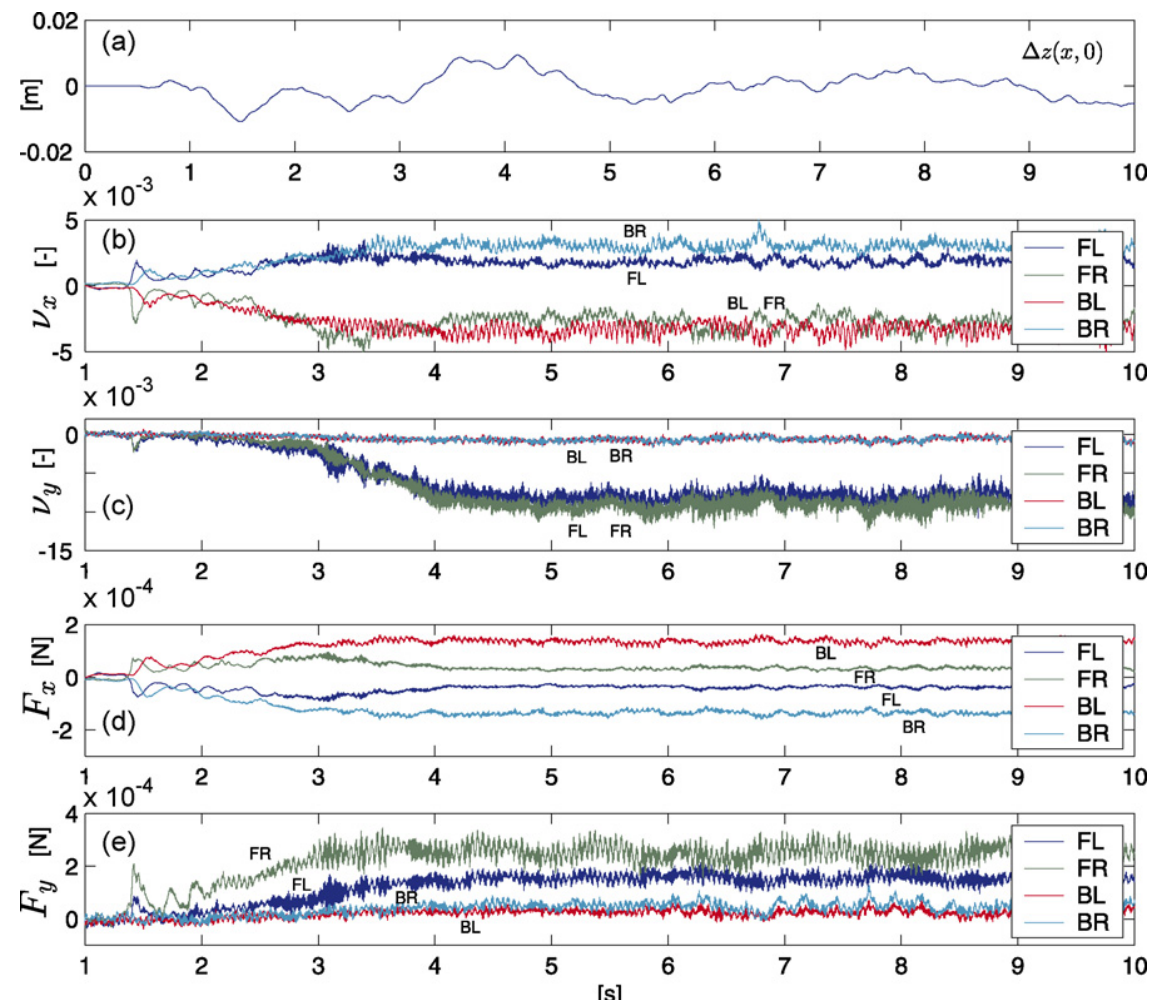

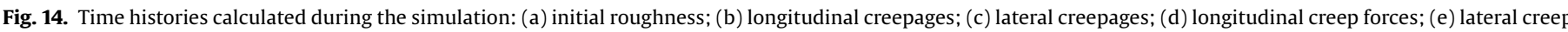
forces. Legend: FL: front left; FR: front right; BL: back left; BR: back right. 


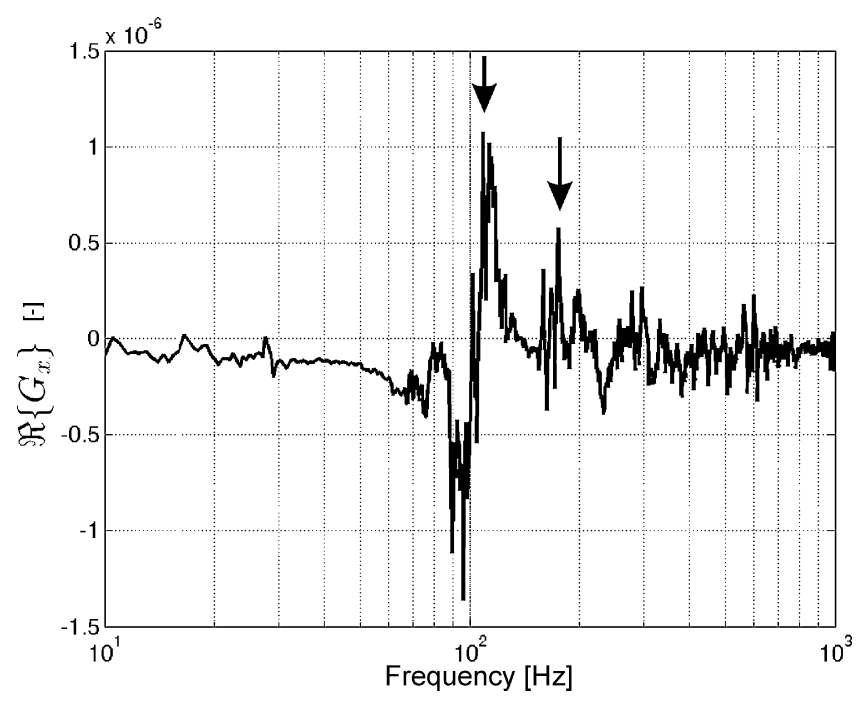

Fig. 15. Longitudinal corrugation growth rate.

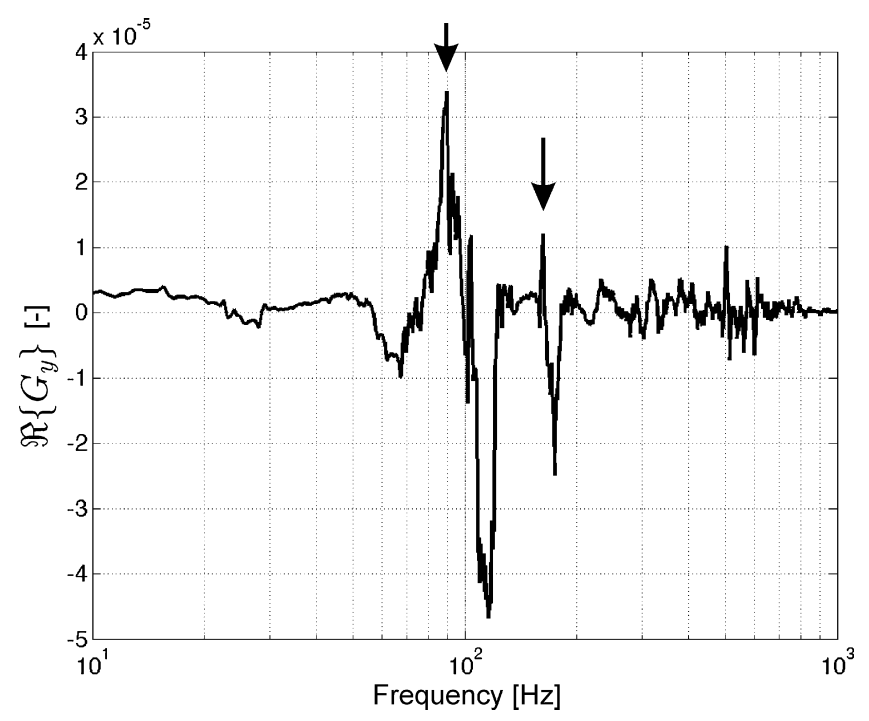

Fig. 16. Lateral corrugation growth rate.

Table 2

Parametric study influence on $G_{y}$

\begin{tabular}{lccc}
\hline Parameter & Variation & 1st peak & 2nd peak \\
\hline Sleeper mass & $\searrow$ & - & $\searrow$ \\
Lateral pad stiffness & $\nearrow$ & - & $\searrow$ \\
Vertical pad stiffness & $\searrow$ & - & $\searrow$ \\
Vehicle speed & $\searrow$ & - & - \\
Superelevation & $\searrow$ & $\searrow$ & $\searrow$ \\
\hline
\end{tabular}

lateral corrugation growth rate are shown because it is one order magnitude higher than the longitudinal one.

Main results for the parameter variations can be summarized as follows:

- Suppressing both the sleeper vertical and lateral resonance has a positive effect on the mitigation of both types of rail corrugation: decreasing the lateral rail pad stiffness, decreasing the vertical rail stiffness and decreasing the mass of the sleeper.

- The influence of the operating condition on rail corrugation was also studied through a variation of the vehicle speed: a decrease of $50 \%$ of the vehicle speed has nearly no effect on the corrugation growth rate.

- A decrease of the superelevation also leads to a better track adaptation and steering capabilities of the vehicle, i.e. a lower wheel load difference and a decrease of the lateral creep forces.

In terms of measures to decrease the growth of rail corrugation, a new solution has been also investigated in this work against the booted sleeper corrugation that has been related to the wavelength of $8 \mathrm{~cm}$. The idea developed was to mount a dynamic vibration absorber on the concrete sleeper, tuned in both vertical and lateral direction. In the vertical direction, it has been tuned to the vertical anti-resonance of the track between the first and the second mode (only the sleeper vibrates); in the lateral direction it has been tuned to the second mode (the rails vibrate in opposite phase with the sleeper). A drawing of the device is proposed in Fig. 17.

Using a ratio between the mass of the absorber and the modal mass of the resonances to damp of $10 \%$, a decrease of $20 \%$ of the corresponding corrugation growth has been found. This limited efficiency is attributed to the high structural damping $(\zeta=0.15)$ of the track modes of interest.

\section{Conclusions}

In this work, frequency and time domain approaches were applied to a corrugated, curved, ballasted track section located in the RER commuter rail network in Paris. In both derivations examined here, firstly the wavelength fixing mechanisms are identified and results from the time and frequency domain approaches are compared through longitudinal and lateral rail corrugation growth rates. The vertical track anti-resonance around $180 \mathrm{~Hz}$ between the first and the second modes of the track and the $\mathrm{P} 2$ resonance are found to be the main cause for corrugation to develop.

It has been shown that, using the frequency domain approach, corrugation associated to the P2 resonance is underestimated, and more accurate predictions are obtained with the non-linear multibody model. An explanation has been given in terms of fundamental difference in the input excitations between these two approaches and the non-linearity of the multi-body model. On the other hand, calculations in the frequency domain are very fast, and the theory
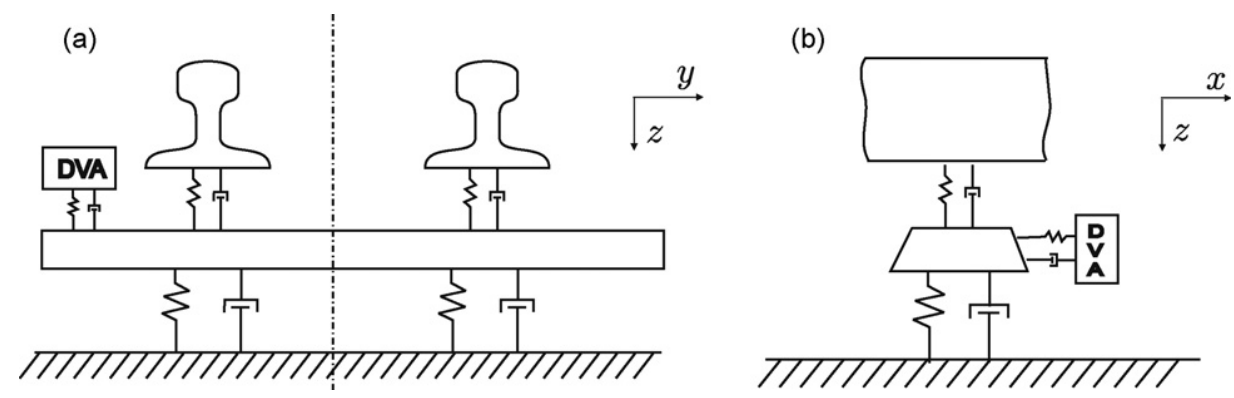

Fig. 17. Dynamic vibration absorber for vertical and lateral sleeper resonances. 
can benefit from a range of tools developed for the study of linear systems, like stability analysis [7,13].

Finally, an extensive parametric study has been performed in order to evaluate the relevance of various parameters on the rail corrugation growth. This made it possible to propose effective measures for mitigating the observed corrugation. More precisely, it has been found that suppressing both the vertical and the lateral sleeper resonance have a positive effect on the mitigation of both types of rail corrugation.

In the same section, a dynamic vibration absorber to decrease the growth of booted sleeper type rail corrugation has been presented. However, if dynamic vibration absorbers are efficient solutions for the decrease of resonances in many cases, it has been found here to show a restricted efficiency due to the high structural damping $(\zeta=0.15)$ of the track modes of interest. Better results could be expected anyway by the use of interesting properties of viscoelastic materials.

\section{Acknowledgments}

This work was performed partly within the European research project "wheel-rail corrugation in urban transport, GRD2-20015006". The authors would like also to thank RATP for supplying the sites.

\section{Appendix A}

The analytical formulations for the longitudinal and lateral corrugation growth rates are given by:

$$
G_{x}(\omega)=-\frac{k_{0}}{2 \rho b V} \frac{\alpha_{x}\left[V v_{x}-j \omega F_{x} R_{x x}\right]}{\begin{array}{c}
\left.R_{z z}+\left(1 / k_{c}\right)+R_{y z}\left(\alpha_{y} V-j \omega \beta_{y} R_{y z} / V+j \omega \beta_{y} R_{y y}\right)\right] \\
\times\left[1+\left(\beta_{x} / V\right) j \omega R_{x x}\right]
\end{array}}
$$

$$
G_{y}(\omega)=-\frac{k_{0}}{2 \rho b V} \frac{\alpha_{y}\left[V v_{y}-j \omega F_{y} R_{y y}-j \omega R_{y z}\left(1+v_{y}\left(\beta_{y} / \alpha_{y}\right)\right)\right]}{\begin{array}{c}
\left.R_{z z}+\left(1 / k_{c}\right)+R_{y z}\left(\alpha_{y} V-j \omega \beta_{y} R_{y z} / V+j \omega \beta_{y} R_{y y}\right)\right] \\
\times\left[1+\left(\beta_{x} / V\right) j \omega R_{x x}\right]
\end{array}}
$$

where $v_{i}$ is the creepage associated to the creep force $F_{i}$ in the direction $i(i=x, y), \alpha_{i}=\partial F_{i} / \partial N_{i}, \beta_{i}=\partial F_{i} / \partial v_{i}, R_{i j}(\omega)$ is the receptance of the coupled vehicle track system between the direction $i$ and $j$ $(i, j=x, y, z)[8]$. This receptance is defined as follows:

$$
R_{i j}(\omega)=R_{i j}^{\mathrm{WHEEL}}(\omega)+R_{i j}^{\mathrm{RAIL}}(\omega)
$$

where $R_{i j}^{\mathrm{WHEEL}}(\omega)$ is the ratio between the displacement of the wheel in the direction $i$ and a point force excitation applied in the direction $j ; R_{i j}^{R A I L}(\omega)$ is the ratio between the displacement of the rail in the direction $i$ and a point force excitation applied in the direction $j$.

\section{References}

[1] RATP, Internal Report.

[2] European Standard, Railway applications - track - acceptance of work. Part 3. Acceptance of rail grinding, milling and planning work in track, January 2006.

[3] C.O. Frederick, A rail corrugation theory, in: Proceedings of the 2nd International Conference of Contact Mechanics and Wear of Rail Systems, 1987, pp. 181-211.

[4] I. Gomez, E.G. Vadillo, An analytical approach to study a special case of booted sleeper track rail corrugation, Wear 251 (2001) 916-924.

[5] I. Gomez, E.G. Vadillo, A linear model to explain short pitch corrugation on rails, Wear 255 (2003) 1127-1142.

[6] I. Gomez, E.G. Vadillo, J. Santamaria, A comprehensive track model for the improvement of corrugation models, J. Sound Vib. 293 (2006) 522-534.

[7] S. Muller, A linear wheel-rail model to investigate stability and corrugation on straight track, Wear 243 (2000) 122-132.

[8] E. Tassilly, N. Vincent, Rail corrugation: analytical model and field tests, Wear 144 (1991) 163-178

[9] E. Tassilly, N. Vincent, A linear model for the corrugation of rails, J. Sound Vib. 150 (1) (1991) 25-45.

[10] K. Hempelmann, K. Knothe, An extended linear model for the prediction of short pitch corrugation, Wear 191 (1996) 161-169.

[11] S. Muller, A linear wheel-track model to predict instabillity and short pitch corrugation, J. Sound Vib. 227 (5) (1999) 899-913.

[12] J.B. Nielsen, Evolution of rail corrugation predicted with a non-linear wear model, J. Sound Vib. 227 (5) (1999) 915-933.

[13] P.A. Meehan, W.J.T. Daniel, T. Campey, Prediction of the growth of wear-type rail corrugation, Wear 258 (2005) 1001-1013.

[14] H. Krause, G. Poll, Wear of the wheel-rail surfaces, Wear 113 (1986) 103-122.

[15] S.L. Grassie, J. Kalousek, Rail corrugation: characteristics, causes and treatments, Proc. Inst. Mech. Eng., J. Rail Rapid Transit. 207 (1993) 57-68.

[16] J.I. Egana, J. Vinolas, M. Seco, Investigation of the influence of rail pad stiffness on rail corrugation on a transit system, Wear 261 (2006) 216-224.

[17] A. Igeland, H. Ilias, Rail head corrugation growth predictions based on nonlinear high frequency vehicle/track interaction, Wear 213 (1997) 90-97.

[18] J.C.O. Nielsen, Numerical prediction of rail roughness growth on tangent tracks, J. Sound Vib. 267 (2003) 537-548.

[19] C. Anderson, A. Johansson, Prediction of rail corrugation generated by threedimensional wheel-rail interaction, Wear 257 (2004) 423-434.

[20] X. Jin, Z. Wen, K. Wang, W. Zhang, Effect of scratch on curved rail on initiation and evolution of rail corrugation, Tribol. Int. 37 (2004) 385-394.

[21] J.J. Kalker, Rolling Contact Phenomena, Chapter Rolling Contact Phenomena, Springer Verlag, 2000, pp. 1-84.

[22] X. Jin, Z. Wen, W. Zhang, Z. Shen, Numerical simutlation of rail corrugation on a curved track, Comput. Struct. 83 (2005) 2052-2065.

[23] X. Jin, Z. Wen, W. Zhang, Z. Shen, Numerical simulation of rail corugation on a curved track, Comput. Struct. 83 (2005) 2052-2065.

[24] Simpack Release 8.7, INTEC GmbH, Wessling, Germany, 2005.

[25] O. Polach, Creep forces in simulations of traction vehicles running on adhesion limit, Wear 258 (2005) 992-1000. 